\title{
On the Tension Between Physics and Mathematics
}

\author{
Miklós Rédei ${ }^{1}$ (D) \\ Published online: 4 February 2020 \\ (C) The Author(s) 2020
}

\begin{abstract}
Because of the complex interdependence of physics and mathematics their relation is not free of tensions. The paper looks at how the tension has been perceived and articulated by some physicists, mathematicians and mathematical physicists. Some sources of the tension are identified and it is claimed that the tension is both natural and fruitful for both physics and mathematics. An attempt is made to explain why mathematical precision is typically not welcome in physics.
\end{abstract}

Keywords Mathematics and physics - Mathematical precision - Mathematical physics · Quantum theory

\section{The "Supermarket Picture" of the Relation of Physics and Mathematics, and Tension of Type I}

According to what can be called the standard picture of the relation of physics and mathematics, physics is a science in the modern sense because it is systematically mathematical, which means two things:

(a) Physics carries out precision measurements aiming at determining values of operationally defined physical quantities. This is what quantitative experimental physics does, and this ensures descriptive accuracy of physics.

(b) Physics sets up mathematical models of physical phenomena that make explicit the functional relationships among the measured quantities; i.e. physics formulates gen-

This paper is a slightly expanded version of the plenary talk delivered at the 2019 Frühjahrstagung (München, March 17-22, 2019) of the Deutsche Physikalische Gesellschaft (DPG) on the invitation of the Arbeitsgruppe Philosophie der Physik of DPG. I wish to thank the representatives of the Arbeitsgruppe Philosophie der Physik, R. Dardashti, M. Kuhlman and C. Wüthrich, for the invitation. Written while staying at the Munich Center for Mathematical Philosophy, Ludwig Maximilians University, supported by the Alexander von Humboldt Foundation and by the National Research, Development and Innovation Office, Hungary, K115593.

Miklós Rédei

m.redei@1se.ac.uk

1 Department of Philosophy, Logic and Scientific Method, London School of Economics and Political Science, Houghton Street, London WC2A 2AE, UK 
eral quantitative physical laws. This is the main activity in theoretical physics and this enables physics to be predictively successful.

Both descriptive accuracy and predictive success should be understood here with a number of qualifications: the descriptive accuracy is never perfect (experimental errors); the operationally defined quantities are not purely empirical (theory-ladenness of observations), natural laws may also refer to entities that are not observable strictly speaking, etc.

While the standard picture of the relation of mathematics and physics as characterized by (a) and (b) captures crucial properties of the role of mathematics in physics, it is somewhat naive because it is (tacitly) based on what I call here the 'supermarket picture' of the relation of mathematics and physics: that mathematics is like a supermarket and physics is a customer. That is to say, it is tacitly assumed that when a physicist needs a mathematical concept, a mathematical structure, or any mathematical tool to formulate the mathematical model of a physical phenomenon, then (s)he just goes to the mathematics-supermarket, looks at the shelves, takes off the product needed, and, possibly after some adjustment and tinkering with it after checkout, (s)he has the mathematical tool suitable to set up the mathematical model.

But this assumption is non-trivial; so non-trivial that it frequently does not hold: mathematical concepts are (frequently) not available in strict, precise, consistent mathematical sense and form at the time needed for physics. Moreover, creating the needed mathematical concepts might take a considerable amount of time. Since physics cannot stand still and wait until the right mathematical concepts have been worked out, physics will necessarily be mathematically sloppy, imprecise, even inconsistent during this time.

One can cite several historical examples to back up this claim. In the next section four (well-known) examples are given. The tension between physics and mathematics arising from the need in physics of some mathematical concepts that are not available at a given time in mathematics I call Tension of type I.

\section{Examples of Tension of Type I}

\subsection{The Missing Derivative in Newton's Mechanics}

Newton would have needed the concept of derivative of a function to formulate the second law of motion. But this concept was not available in mathematics in precise form until the work of Cauchy and Weierstrass in the nineteenth century. So Newton did what he could: reasoning in an inconsistent manner involving infinitesimals (quantities that were assumed to have both finite and zero value) to calculate derivatives. To be more precise, Newton used both algebraic and geometric methods in his calculations. The algebraic one relied on the notion of infinitesimals; the geometric reasonings used "ultimate ratios of vanishing quantities" (Guicciardini 2002, 320). The algebraic calculations were not published until after the Principia, in which geometric reasonings dominate. Logical problems with both reasonings had been pointed out already in Newton's time:

Berkeley was to observe in the Analyst (1734) that the "limits of vanishing quantities" employed in the Principia are as mysterious as the infinitesimals, since the "ultimate ratio of two vanishing quantities", when the quantities are "vanished" is $0 / 0$, and before they have vanished the ratio is not the "ultimate". (Guicciardini 2002, 320) 
Concerning the reasoning in terms of infinitesimals, Berkeley writes in The Analyst (1734):

For when it is said, let the increments vanish, i.e. let the increments be nothing, or let there be no increments, the former supposition that the increments were something, or that there were increments, is destroyed, and yet a consequence of that supposition, i.e. an expression got by virtue thereof, is retained. Which, by the foregoing lemma, is a false way of reasoning. [...]

[The foregoing Lemma:]

'If with a view to demonstrate any proposition, a certain point is supposed, by virtue of which certain other points are attained; and such supposed point be it self afterwards destroyed or rejected by a contrary supposition; in that case, all the other points attained thereby, and consequent thereupon, must also be destroyed and rejected, so as from thence forward to be no more supposed or applied in the demonstration.' This is so plain as to need no proof. (Berkeley 2002, 5-6)

It should be noted that, while it is standard view in history of mathematics that Newton did not have a consistent concept of limit and only since Cauchy's work is this notion available in precise ('epsilon-delta') form, the standard view is contested by B. Pourciau (2001), who claims that Newton had a very clear concept of limit. It is incontestable however that the concept of derivative had not been available in the mathematics supermarket when it was needed for mechanics, and Newton was struggling with creating it.

\subsection{Missing Spectral Theory of Selfadjoint Operators in Quantum Mechanics Before von Neumann}

Hilbert space quantum theory was established systematically in a mathematically precise form by von Neumann, and was summarized in his book (von Neumann 1932a). The book was based on three foundational papers, von Neumann (1927a; 1927b; 1927c), all published in 1927 (they were communicated by M. Born in May and in November of 1927). Of these three papers the first von Neumann (1927a) introduced the notion of abstract Hilbert space and treated the 'eigenvalue problem' of (possibly unbounded) selfadjoint operators having possibly a continuous part in their spectrum in a mathematically consistent manner, without using the Dirac delta function. The technically complete, full analysis of the spectral theorem was worked out by von Neumann in a substantial paper published in 1930 only (von Neumann 1930) (the paper was received by the journal on December 15, 1928). These mathematically precise papers had been preceded by a paper co-authored by Hilbert et al. (1928) (received by Mathematische Annalen on April 6, 1927). This latter paper is the first attempt to systematize (axiomatize) standard Hilbert space quantum mechanics, and the paper is based on Hilbert's lecture on foundations of quantum mechanics in 1926. Although the paper was published in a mathematics journal, the authors are aware that the calculations they present there do not meet the criteria of precise mathematics:

From a mathematical point of view the method of calculation is rather unsatisfactory because one is never certain whether the operations involved are really admissible. For this reason we do not detail them further. But we hope to return to these issues on another occasion. (Hilbert et al. 1928, 30, my translation ${ }^{1}$ )

\footnotetext{
1 German original: "Vom mathematischen Standpunkt ist aber die dabei eingeschlagene Rechnungsweise [...] ziemlich unbefriedigend, da man nie sicher ist, in welchem Umfange die dabei auftretenden Operationen wirklich zulässig sind. Deshalb sehen wir zunächst von der weiteren Ausführung dieser Gedanken ab. Wir hoffen jedoch, bei anderer Gelegenheit auf diese Fragen zurückkommen zu können.”
} 
And then they refer to von Neumann's forthcoming paper (von Neumann 1927a) in which the problem is treated in a mathematically general and precise manner.

The point in this example is this: until the publication of von Neumann's two foundational papers von Neumann (1927a) and von Neumann (1930) the 'eigenvalue problem' could not be (and was not) treated in physics in a mathematically precise manner. Quantum mechanics was thus mathematically sloppy until the publication of these papers: the notion of spectrum was foggy, assumptions were made that were false (that every element in the spectrum is an eigenvalue), and the relation of spectrum and the operator was unclear.

One might regard the mathematical clarification of the concept of derivative far more significant than the tidying up the concept of spectrum of a selfadjoint operator on a Hilbert space-after all, the notion of derivative became indispensable in every corner of physics. Assessing the relative importance of mathematical clarifications of concepts used in (possibly different subfields of) physics seems to be an interesting but very subtle issue. Subtle because it is likely that the relative importance is changing as physics is developing and the development is unpredictable. The analysis gets even more complicated if one tries to compare the importance of events that are separated by a large time interval-like the clarification of the derivative and of the spectrum. But such an analysis is not attempted here. In particular, I do not claim that making the spectrum mathematically precise was just as important as clarifying the concept of derivative. One should however realize that the clarification of the spectrum was highly significant when viewed 'internally' from the perspective of quantum theory because it concerned a central assumption in quantum mechanics: that the possible values of observables are elements of the spectrum of the operators representing the observables, and it was a great achievement of the theory that it could describe mathematically the empirically observed spectrum of the hydrogen atom.

\subsection{Missing Ergodic Theory in Classical Statistical Mechanics Before von Neumann}

The concept of ergodicity goes back to the work of Boltzmann in the second half of the nineteenth century. Ergodicity of the dynamical system describing the time evolution of the physical system of many particles moving according to the laws of motion of classical mechanics was supposed to ensure that the time averages of macroscopic observables are equal to the phase averages of these observables. But making this idea mathematically precise turned out to be extremely challenging:

It took quite a time until the mathematical object of the ergodic hypothesis was found. Indeed only in 1929, Koopman (1931), began to investigate groups of measure-preserving transformations of a measure space, or in other language, groups of unitary operators in a Hilbert space. (Szász 1996, 3)

Koopman's idea ('Koopmanism') enabled one to investigate the problem of ergodicity of dynamical systems in terms of functional analysis, and it was in this formalism that the first rigorous mathematical result ('Mean Ergodic Theorem') was obtained by von Neumann (1932b): he proved that the time average exists in the topology defined by the Hilbert space norm. von Neumann's work also made clear that the concept of ergodicity needed for the equality of time and phase averages is metric transitivity: the condition that there exist no measurable subsets of the constant energy hypersurface that are invariant with respect to the dynamic and which have non-trivial measure (Mackey 1990). Thus it became conceptually clear that for the foundations of classical statistical mechanics one should prove that 
the dynamical systems are ergodic in the sense of metric transitivity. But proving metric transitivity also turned out to be an extremely difficult problem:

After more than one hundred years, ergodicity is still not established in the simplest mechanical model, in the system of elastic hard balls though I expect we are not far from a solution. But as to generic interactions, even the questions are not clearly posed and it might well be that there will not be a final understanding after the next hundred years either. (Szász 1996, 19)

It should be noted that it is controversial whether the equality of time and phase space averages along the lines of ergodicity is indeed crucial for the foundations of classical statistical mechanics (Bricmont 1995; Earman and Rédei 1996). The point by recalling this episode is not that I wish to defend the significance of ergodicity for classical statistical mechanics but to illustrate that to the extent this notion was thought in physics to be important before the concepts of dynamical systems and of the notion of ergodicity became precise, physics was sloppy and foggy, and that it took a very long time before the mathematically acceptably clear and physically relevant concepts have been found.

\subsection{Missing Operator Valued Tempered Distribution in Quantum Field Theory Before Wightman}

Presence of mathematical inconsistencies in relativistic quantum field theory were very well known after the first attempts to quantize fields had been made; the inconsistency is explicitly mentioned by R.F. Streater and A.S. Wightman:

the quantum theory of fields never reached a stage where one could say with confidence that it was free from internal contradictions-nor the converse. In fact, the Main Problem of quantum field theory turned out to be to kill it or cure it: either to show that the idealizations involved in the fundamental notions of the theory are incompatible in some physical sense, or to recast the theory in such a form that it provides a practical language for the description of elementary particle dynamics. (Streater and Wightman 1964, 1, emphasis added)

Wightman's solution of the inconsistency problem was to follow Hilbert's suggestion in his '6th problem' (Hilbert 1983; Wightman 1983; Rédei 2014). Hilbert asked for the axiomatization of physical theories and this is what Wightman did in quantum field theory. In this axiomatization $^{2}$ (summarized in Streater and Wightman (1964); for a compact presentation see Reed and Simon (1975, Sect. IX.8)) Wightman (i) separated mathematical model and physical interpretation of mathematical model sharply and explicitly; (ii) stipulated mathematical axioms for the mathematical model; (iii) formulated the task of showing that the axioms are consistent by creating models. The crucial mathematical concept in terms of which the axiomatization was carried out was the notion of smeared field: the assignment of an (unbounded) operator to the functions in the Schwartz space of rapidly decreasing smooth functions on which tempered distributions are defined. One of the axioms required that the expectation values of the operators, viewed as maps from the Schwartz space, be

\footnotetext{
2 The axiomatization was formulated in a joint paper by Wightman and Gårding (1964) and thus it is sometimes called Wightman-Gårding axiomatization; however, the shorter name 'Wightman axiomatization' has become standard and I follow this terminology.
} 
tempered distributions in the sense of distribution theory established by L. Schwartz. This was in sharp contrast to the naive idea (which was a source of inconsistency) that a quantum field theory assigns operators to singular spacetime points. Treating quantum fields as operator valued tempered distributions allowed proving that the axioms are consistent by constructing models describing free (non-interacting) quantum fields (see Sect. X.7 in Reed and Simon (1975) for a summary).

From the perspective of Tension of type I the point in this example is this: the concept of quantum field as operator valued tempered distribution that is needed for a mathematically consistent model of quantum field theory had not been available in the mathematics supermarket at the time it was needed for physics, i.e. when first attempts were made to quantize fields in the 1920s. Moreover, this concept could not have been created before the mathematically precise notion of distribution was created by Schwartz in the 1940s: Schwartz published the theory in four papers in the period 1945-1949, summarized the theory in his two-volume book (Schwartz 1950-1951), and it is known that he had the concept of tempered distribution in 1947 (Lützen 1982, 157).

It should be noted that this example has not only historical interest, it is very relevant form the perspective of today's physics: constructing mathematically precise models of interacting quantum fields in $(3+1)$ dimension is still an open problem:

Despite these successes [of the Standard Model], however, establishing the Standard Model (or part of it) as a mathematically complete and consistent quantum field theory remains an unsettled challenge. (Fredenhagen et al. 2007, 62)

Though it [QFT] has undergone a long and complex development from its origins [...] and has attained an ever increasing theoretical sophistication, it is still not clear in which sense the physically central quantum field theories such as quantum electrodynamics (QED), quantum chromodynamics (QCD) and the Standard Model (SM) are mathematically well defined theories based upon fundamental physical principles that go beyond the merely ad hoc. (Summers 2012,1)

\section{Tension of Type I Drives Mathematical Developments}

Many other examples for Tension of type I could be found in the history of physics, even in contemporary physics (path integral formulations in quantum theory, renormalization group techniques in quantum field theory and statistical physics). This indicates that the phenomenon is not unusual, happens regularly and belongs to the normal mode of how physics develops. Moreover, the tension between physics and mathematics caused by missing mathematical concepts and the presence of the contradictions drives the development in mathematics as well. This idea was formulated by von Neumann:

The most vitally characteristic fact about mathematics is, in my opinion, its quite peculiar relationship to the natural sciences, or, more generally, to any science which interprets experience on a higher than purely descriptive level. [...] It is undeniable that some of the best inspirations in mathematics-in those parts of it which are as pure mathematics as one can imagine-have come from the natural sciences. (von Neumann 1961, 4, emphasis added)

But von Neumann is not the only mathematician taking this position. More recent formulations of the same idea (with emphasis on specific fields) can be found in the replies of 
prominent mathematicians to the paper by Jaffee and Quinn (1993). Here are two characteristic views:

D. Ruelle:

The importance for pure mathematics of ideas coming from theoretical physics is of course well known [...] the physical ideas of equilibrium statistical mechanics have richly contributed to the mathematical theory of smooth dynamical systems (with the concepts of entropy, Gibbs states, etc., see my note in BAMS(NS) 19 (1988), 259 268). (Atiyah et al. 1994, 197)

K. Uhlenbeck:

The relationship between physics and mathematics has been fundamental to both for a long time. The gap between the two is significant primarily in this century, as pure mathematics became very abstract, experimental physics became very expensive, and the world became more complicated. However, even through this century, mathematics has relied on physics for input quite steadily. [...] I am sure other replies will point out the influence of mechanics on calculus, optics on Riemannian and symplectic geometry, general relativity on differential geometry, quantum mechanics on functional analysis, geometric optics on harmonic analysis, and gauge theory on four-manifold topology. (Atiyah et al. 1994, 202)

A more recent formulation of the idea of physics motivating research in pure mathematics can be found in Dijkgraaf (2017).

Accepting von Neumann's (quasi-empiricist) position about mathematics has consequences: one is that it is perfectly understandable that mathematical concepts 'apply effectively' in physics. This is in sharp contrast to the position von Neumann's colleague Wigner takes: the applicability of mathematics in physics has been famously declared by Wigner to be a mystery, something which we cannot explain rationally and which we do not deserve (Wigner 1960, 223, 237). But the effectiveness of mathematics in physics becomes mysterious only if one sees mathematics as emerging in a process that is cut off from empirical science-as Wigner sees this, in sharp contrast to von Neumann:

it is unquestionably true that the concepts of elementary mathematics and particularly elementary geometry were formulated to describe entities which are directly suggested by the actual world, the same does not seem to be true of the more advanced concepts, in particular the concepts which play such an important role in physics. (Wigner 1960, 2, emphasis added)

The examples in Sect. 2 illustrate that not only the concepts of elementary mathematics originate in the empirical sciences but also the advanced ones-just as von Neumann claims - in contrast to Wigner.

Wigner's claim about the mysteriously effective applicability of mathematics in physics has been analyzed in a number of papers. Here I only comment on some of the most recent works that discuss Wigner's position.

Islami (2017, 4849-4850; 2018, 1338) and Ferreirós (2017) point out that Wigner's position about mathematics is close to that of a formalist. Ferreirós (2017) isolates sources of formalist influence on Wigner (including ones rooting in the general intellectual climate of the 1920s). There also seem to be strong arguments in favour of this formalist position. One, mentioned by Wigner, is the creation of complex numbers, which, as the argument goes, had been introduced into mathematics long before they found their application in physics (for instance in quantum theory). A careful look at the complex history of 
the creation of complex numbers and of their application in physics shows however that the applicability is perfectly reasonable and not a mystery; hence cannot be regarded as a strong argument in support of Wigner's position (see Islami (2018) for details).

According to the diagnosis of Islami and Wiltsche,

[a]t the heart of applicability problem lies what we call the distinctness thesis, i.e. the thesis that mathematics and the physical sciences are categorically distinct. (Islami and Wiltsche 2019, 3)

Once the distinctiveness is assumed, it is difficult to come up with an explanation of the effectiveness and applicability of mathematics in physics. But it is not obvious how one can establish a positive non-distinctiveness position (von Neumann did not try to articulate one). Islami and Wiltsche (2019) attempt to work out such a position by making heavy use of concepts provided by phenomenology. Their main conclusion is that "[i]n the case of modern theoretical physics [...] there is no such distinctness: the objects of physics are constituted mathematically" (Islami and Wiltsche 2019, 19). In fact, Islami interprets Wigner's own views on symmetry principles as a kind of solution to the applicability problem in the spirit of the idea that mathematics is constitutive of modern theoretical physics: "The invariance principles give laws their universality, a feature that makes their mathematical formulation possible" (Islami 2017, 4857).

In a recent paper Grinbaum (2019) also argues that effectiveness of mathematics in physics is perfectly reasonable and not mysterious in cases when mathematics serves in a regulative role that guides and constrains theory building in physics in situations when physics is not (yet) in the position of formulating natural laws based on detailed understanding of phenomena.

The other consequence is that, since the creation of rigorous, well-defined mathematical concepts motivated by physics is not a simple process, it can happen that developing the mathematical concepts motivated by physics takes a long time. During this time not only physics is left without a proper mathematical concept but even mathematics can appear imprecise and foggy.

\section{Absence of Rigour in Mathematics?}

But can mathematics itself be non-rigorous, imprecise, non-exact? This is controversial but many mathematicians have taken the position that rigour, precision and exactness are not binary and are not absolute notions but come in different forms, in different degrees, in different contexts and they change over time. This is again a position that von Neumann took:

it is hardly possible to believe in the existence of an absolute, immutable concept of mathematical rigour, dissociated from all human experience. I am trying to take a very low-brow attitude on this matter. Whatever philosophical or epistemological preferences anyone may have in this respect, the mathematical fraternities' actual experiences with its subject give little support to the assumption of the existence of an a priori concept of mathematical rigour. (von Neumann 1961, 6)

Other similar expressions:

M. Atiyah:

The history of mathematics is full of instances of happy inspiration triumphing over a lack of rigour. Euler's use of wildly divergent series or Ramanujan's insights are 
among the more obvious [...] The marvelous formulae emerging at present from heuristic physical arguments are the modern counterparts of Euler and Ramanujan, and they should be accepted in the same spirit of gratitude tempered with caution. (Atiyah et al. 1994, 178)

R. Thom:

I do still believe that rigor is a relative notion, not an absolute one. It depends on the background readers have and are expected to use in their judgment. Since the collapse of Hilbert's program and the advent of Gödel's theorem, we know that rigor can be no more than a local and sociological criterion. (Atiyah et al. 1994, 203)

Atiyah and Thom express different sources of absence of rigor in mathematics: Atiyah emphasizing the useful, positive role of heuristic, whereas Thom points out the negative consequences of axiomatism that is subject of the incompleteness results in logic. While Thom's declaring rigour as nothing more than a social criterion seems too radical, both Atiyah and Thom display a relaxed attitude towards rigour. This leaves ample room for the phenomenon of extended periods of searching for precise mathematical concepts motivated by the problem situation in physics during which lack of rigour is tolerated in mathematics.

\section{Mathematical Precision Is Not Welcome in Physics by Physicists- Tension of Type II}

Even when precise mathematical concepts are available to use in theoretical physics, physicists typically do not aim at mathematical precision. Some regard it as not useful, or irrelevant, or illusory, or impossible; some explicitly reject it as unimportant. I call the tension arising from physicists' dismissive attitude towards mathematical precision Tension of type II. Below are quotations as evidence for the presence of this kind of tension between mathematics and physics.

\section{A. Jaffee and F. Quinn:}

The typical attitude of physicists toward mathematics is illustrated by a passage from a book of P. W. Anderson, "We are talking here about theoretical physics, and therefore of course mathematical rigor is irrelevant and impossible." (Jaffee and Quinn $1993,5)^{3}$

Needless to say, Jaffe and Quinn, being mathematical physicists, do not share this attitude. But the attitude is indeed there, as the following quotation from R. Feynman also shows:

The mathematical rigor of great precision is not very useful in physics. But one should not criticize the mathematicians on this score [...] They are doing their own job. (Feynman 1965, 56)

Another mathematical physicist, W. Thirring, recalls the following episode:

\footnotetext{
3 Jaffee-Quinn's footnote: "This is attributed by Anderson to Landau. Anderson continues, 'This is not quite so, but it is very close to it.' However, he revealingly remembered the passage incorrectly; it reads (Landau and Lifshitz 1938), 'No attempt has been made at mathematical rigor in the treatment, since this is anyhow illusory in theoretical physics [...]'” (Jaffee and Quinn 1993, 5).
} 
For a long time his [von Neumann's] importance for physics was underrated, Pauli once told me that he had said to v. Neumann: "If a mathematical proof is what matters in physics you would be a great physicist". I disagree with this statement, I think he had the right vision of what will become important in physics. (Thirring 2001, 5)

That mathematical precision is somewhat alien in physics is reflected by the controversial status of mathematical physics as a discipline: mathematical physicists find themselves between two chairs, as scholars who are doing something non-standard, weird, something that is not really appreciated either by mathematicians or by physicists, and frequently they are on the defensive. This feeling is reflected by Wightman's remark:

The physicists who have engaged in this kind of work [doing axiomatic, constructive QFT] are sometimes dubbed the Veldferein [sic!]. Cynical observers have compared them to the Shakers, a religious sect of New England who built solid barns and led celibate lives, a non-scientific equivalent of proving rigorous theorems and calculating no cross sections. (Streater and Wightman 1964, 1)

Given that the Shakers, due to the celibacy they adopted, have become nearly extinct ${ }^{4}$, Wightman effectively regards mathematical physicists as an endangered species. Fortunately, mathematical physics has not faded away as a discipline. Quite on the contrary: it has established itself institutionally. Proofs of this include: Founding (in 1976) of the International Association of Mathematical Physics; the flourishing of respected journals specializing in mathematical physics; the organization of regular conferences devoted to mathematical physics; and a visible and active scientific community. But Wightman's words express the feeling that pursuing mathematical physics is a scientific activity that is more problematic socially than doing simple physics or mathematics.

\section{Why Physicists Do Not Appreciate Mathematical Precision}

There are two, intertwined intuitions in the dismissive attitude towards mathematical precision in physics: a good one and a problematic one.

The good intuition in rejecting mathematical precision is that striving for mathematical precision is not the main, not the decisive source of development of physics: experimenting and observations are.

The problematic part of the intuition is to think that mathematical precision does not contribute to the development of physics hence it is not useful epistemologically. But striving for mathematical precision does contribute to the development of physics and making explicit this contribution serves as an argument against the claim of uselessness and irrelevance of mathematical rigour in physics.

First of all, mathematical precision forces conceptual precision and clarity, which is coupled with and amplified by critical self-reflection. This feature of mathematical physics is nicely formulated by von Neumann in his 1949 letter to Cirker, head of Dover publishing house, with whom von Neumann apparently discussed a possible publication of an English translation of his book von Neumann (1932a) (see Rédei 2005, 286-287). In this letter von

\footnotetext{
4 "Before the Civil War there were nearly 6000 Shakers in 23 communities. Today, there is just one active Shaker village left, with just three members" (Abernethy 2010, 1).
} 
Neumann characterizes the nature of his book, which is the paradigm example of a work in mathematical physics:

The subject-matter is partly physical-mathematical, partly, however, a very involved conceptual critique of the logical foundations of various disciplines (theory of probability, thermodynamics, classical mechanics, classical statistical mechanics, quantum mechanics). This philosophical-epistemological discussion has to be continuously tied in and quite critically synchronised with the parallel mathematical-physical discussion. It is, by the way, one of the essential justifications of the book, which gives it a content not covered in other treatises, written by physicists or by mathematicians, on quantum mechanics. (von Neumann to Cirker, October 3, 1949) (Rédei 2005, 92)

Mathematical precision and explicitness also requires to make the link between the mathematical model and physical reality explicit and unambiguous. This effect of mathematical precision can expose serious conceptual-interpretational difficulties even in a mathematically impeccable physical theory. This contributes to a much better understanding of the theory and can pave the way to new theories. An example of this is von Neumann's tidying up mathematically the Hilbert space formalism of quantum mechanics and his subsequent abandoning it: soon after the Hilbert space formalism had been worked out precisely by him, and to a large extent due to the fact that the basic principles of quantum mechanics have been clarified in a mathematically precise way, difficulties with the interpretation from the perspective of the frequency interpretation of quantum probability became visible. von Neumann had hoped to get around the difficulties by suggesting the theory of type $I I_{1}$ factor von Neumann algebras as the suitable framework for quantum theory-together with a logical interpretation of quantum probability replacing the frequency view. (See the papers Araki (1990), Rédei (1996; 2001), Rédei and Stöltzner (2006), Valente (2008) for the details of this story, including the discussion of why von Neumann's suggestion was unsatisfactory.)

Mathematical precision, especially if it is realized in an axiomatic formulation of a physical theory, also makes explicit the conceptual structure and logical dependencies within a theory. Constructive (axiomatic) quantum field theory illustrates this nicely, and this has also been observed in connection with axiomatic reconstructions of non-relativistic quantum mechanics (Rovelli 1996; Grinbaum 2007). Mathematical precision also contributes to the clarification of relation of different physical theories-ergodic theory is an example of this effect of mathematical precision. These are also instances of how mathematical precision leads to conceptual precision.

Forcing conceptual precision, requiring unambiguous relation of mathematics and physical reality, clarifying intra- and intertheoretic relations of physical theories, and thereby exposing possible conceptual limitations and difficulties, which can in turn lead to the development of new theories - these are epistemic virtues different from (and in addition to) descriptive accuracy and predictive success. Given that striving for mathematical precision in physics facilitates the realization of these epistemic values, mathematical precision has a positive epistemic contribution to physics.

But acknowledging that mathematical precision is useful epistemologically is not easy for psychologically-flavoured reasons that are intimately related to the specific way mathematical precision is epistemologically useful: mathematical precision forces conceptual precision and clarity. But conceptual precision makes it also clear what is not clear conceptually at a given time in physics. Thus mathematical precision, through forcing conceptual precision, exposes the fundamental limits of a physical theory at a given time. Since at that time it might not be possible to go beyond those limits by creating new and better physical 
theories, this is an epistemologically frustrating situation. This can be frustrating psychologically: it can be perceived by physicists as limits of their own, subjective capabilities (cleverness, ingenuity, creativity, intellectual power). This is unpleasant, can be experienced even as an existential threat and it is understandable that physicists do not wish to experience it. ${ }^{5}$

\section{Concluding Comments}

The examples in Sect. 2 illustrate not only the presence of Tension of type I but also an important feature of this tension: the mathematical imprecision in physics causing Tension of type I is not an 'anything goes' kind of uncontrolled irrationality but a 'disciplined sloppiness': Newton's reasoning about derivatives, however objectionable from the perspective of strict logic, did have a rich, positive content that could be articulated subsequently with precision; Boltzmann's deep intuition about the stochastic behaviour of classical mechanical dynamical systems was an insight with rich informal content that found its precise expression in terms of measure theory and advanced calculus; and the physical intuition guiding quantization of fields could be given an axiomatic formulation that could be shown consistent by displaying models describing free quantum fields. On the basis of these successes one also can expect that heuristic quantum field theory's currently mathematically objectionable treatment of interacting quantum fields will eventually find a form that is mathematically unobjectionable. The current situation in heuristic quantum field theory might thus be analogous to the one in Newton's time when the reasoning involving derivatives was formally objectionable but contentually correct. ${ }^{6}$

Mathematical precision can play an especially important role in certain fields in current physics: in areas where theoretical physics has become unusually speculative and has got very far away from empirical testability. When empirical testability is out of sight, an important regulative principle of theory construction can be mathematical precision. ${ }^{7}$

Thus the presence of different type of tensions between physics and mathematics is not the sign of trouble but a sign of good health of both disciplines.

Acknowledgements Funding was provided by Humboldt Foundation (Grant No. 1168469 - GBR - CFS) and National Research, Development and Innovation Office (Grant No. K115593).

Open Access This article is licensed under a Creative Commons Attribution 4.0 International License, which permits use, sharing, adaptation, distribution and reproduction in any medium or format, as long as you give appropriate credit to the original author(s) and the source, provide a link to the Creative Commons licence, and indicate if changes were made. The images or other third party material in this article are included in the article's Creative Commons licence, unless indicated otherwise in a credit line to the material. If material is not included in the article's Creative Commons licence and your intended use is not permitted by statutory regulation or exceeds the permitted use, you will need to obtain permission directly from the copyright holder. To view a copy of this licence, visit http://creativecommons.org/licenses/by/4.0/.

\footnotetext{
5 An anonymous referee pointed out that other psychological factors (such as physicists finding mathematical precision simply boring) also should be taken into account in explaining why physicists do not welcome mathematical precision in physics. It is indeed very likely that a number of different psychological factors play a role in shaping the attitude of physicists towards mathematical precision. It might be interesting to aim at a more complete analysis of this psychological—as opposed to epistemological-aspect of the issue.

${ }^{6}$ C. Wüthrich's comment in the discussion after the talk.

7 This idea was mentioned by K.H. Rehren in the discussion after the talk, and is in the spirit of the reasoning in Grinbaum (2019).
} 


\section{References}

Abernethy, B. (2010). The shakers. Religion \& Ethics Newsweekly, September. https://www.pbs.org/wnet/ religionandethics/2011/03/18/september-17-2010-the-shakers/7026/. Accessed April 8, 2019.

Araki, H. (1990). Some of the legacy of John von Neumann in physics: Theory of measurement, quantum logic and von Neumann algebras in physics. In J. Glimm, J. Impagliazzo, \& I. Singer (Eds.), The Legacy of John von Neumann (Proceedings of symposia in pure mathematics, Vol. 50, pp. 136-139). Providence, RI: American Mathematical Society.

Atiyah, M., et al. (1994). Responses to: A. Jaffe and F. Quinn, "Theoretical mathematics: toward a cultural synthesis of mathematics and theoretical physics" [Bull. Amer. Math. Soc. (N.S.) 29 (1993), no. 1, 1-13; MR1202292 (94h:00007)]. Bulletin (New Series) of the American Mathematical Society, 30(2), $178-207$.

Berkeley, G. (2002). The analyst; or a discourse addressed to an infidel mathematician. Wherein it is examined whether the object, principles, and inferences of the modern analysis are more distinctly conceived, or more evidently deduced, than religious mysteries and points of faith. Dublin 1734: S. Fuller. The modern edition prepared by D.R. Wilkins (2002, Dublin) and available in pdf format from https:// www.maths.tcd.ie/pub/HistMath/People/Berkeley/Analyst/Analyst.pdf is used for this paper. Accessed April 13, 2019.

Bricmont, J. (1995). Science of chaos or chaos in science? Physicalia Magazine, 17(3-4), 159-208.

Dijkgraaf, R. (2017). Quantum questions inspire new math. Quantamagazine. https://www.quantamagazine. org/how-quantum-theory-is-inspiring-new-math-20170330/. Accessed May 30, 2019.

Earman, J., \& Rédei, M. (1996). Why ergodic theory does not explain the success of equilibrium statistical mechanics. The British Journal for the Philosophy of Science, 47(1), 63-78.

Ferreirós, J. (2017). Wigner's "Unreasonable effectiveness" in context. Mathematical Intelligencer, 39(2), 64-71.

Feynman, R. (1965). The character of physical law. Cambridge, MA: MIT Press.

Fredenhagen, K., Rehren, K. H., \& Seiler, E. (2007). Quantum field theory: Where we are. In I. O. Stamatescu \& E. Seiler (Eds.), Approaches to fundamental physics. An assessment of current theoretical ideas (Lecture notes in physics, Vol. 721, pp. 61-87). Berlin: Springer.

Grinbaum, A. (2007). Reconstruction of quantum theory. The British Journal for the Philosophy of Science, $58(3), 387-408$.

Grinbaum, A. (2019). The effectiveness of mathematics in physics of the unknown. Synthese, 196(3), 973-989.

Guicciardini, N. (2002). Analysis and synthesis in Newton's mathematical work. In I. B. Cohen \& G. E. Smith (Eds.), The Cambridge companion to Newton (pp. 308-328). Cambridge: Cambridge University Press.

Hilbert, D. (1983). Mathematical problems. Lecture delivered before the International Congress of Mathematicians at Paris in 1900. In F. E. Browder (Ed.), Mathematical developments arising from Hilbert Problems (Proceedings of symposia in pure mathematics, Vol. 28, pp. 1-34). Providence, RI: American Mathematical Society.

Hilbert, D., von Neumann, J., \& Nordheim, L. (1928). Über die Grundlagen der Quantenmechanik. Mathematische Annalen, 98, 1-30. Received on April 4, 1927. (Reprinted in A. H. Taub (Ed.). John von Neumann: Collected works. Vol. 1: Logic, theory of sets and quantum mechanics, Oxford/London/ New York/Paris, 1961: Pergamon Press.) No. 7.

Islami, A. (2017). A match not made in heaven: On the applicability of mathematics in physics. Synthese, 194, 4839-4861.

Islami, A. (2018). A complex problem for formalists. Journal of Applied Logic. The IfCoLog Journal of Logics and their Applications, 5(6), 1337-1364.

Islami, A., \& Wiltsche, H. A. (2019). A match made on earth on the applicability of mathematics in physics. In P. Berghofer \& H. A. Wiltsche (Eds.), Phenomenological Approaches to Physics (Synthese library, pp. 1-21). Berlin: Springer.

Jaffee, A., \& Quinn, F. (1993). Theoretical mathematics: Toward a cultural synthesis of mathematics and theoretical physics. Bulletin (New Series) of the American Mathematical Society, 29(1), 1-13.

Koopman, B. O. (1931). Hamiltonian systems and transformations in Hilbert space. Proceedings of the National Academy of Sciences of the United States of America, 17(5), 315-318.

Landau, L., \& Lifshitz, E. (1938). Statistical physics. London: Oxford University Press.

Lützen, J. (1982). The prehistory of the theory of distributions (Studies in the history of mathematics and physical sciences, Vol. 7). New York: Springer. 
Mackey, G. W. (1990). Von Neumann and the early days of ergodic theory. In J. Glimm, J. Impagliazzo, \& I. Singers (Eds.), The legacy of John von Neumann (Proceedings of symposia in pure mathematics, Vol. 50, pp. 25-38). Providence, RI: American Mathematical Society.

Pourciau, B. (2001). Newton and the notion of limit. Historia Mathematica, 28(1), 18-30.

Rédei, M. (1996). Why John von Neumann did not like the Hilbert space formalism of quantum mechanics (and what he liked instead). Studies in History and Philosophy of Science Part B: Studies in History and Philosophy of Modern Physics, 27(4), 493-510.

Rédei, M. (2001). Von Neumann's concept of quantum logic and quantum probability. In M. Rédei \& M. Stöltzner (Eds.), John von Neumann and the foundations of quantum physics. Institute Vienna Circle Yearbook (pp. 153-172). Dordrecht: Kluwer Academic Publishers.

Rédei, M. (Ed.) (2005). John von Neumann: Selected letters (History of mathematics, Vol. 27). Rhode Island: American Mathematical Society and London Mathematical Society.

Rédei, M. (2014). Hilbert's 6th problem and axiomatic quantum field theory. Perspectives on Science, 22(1), 80-97.

Rédei, M., \& Stöltzner, M. (2006). Soft axiomatization: John von Neumann on method and von Neumann's method in the physical sciences. In E. Carson \& R. Huber (Eds.), Intuition and the axiomatic method (Western Ontario series in philosophy of science, Vol. 70, pp. 235-249). Dordrecht: Springer.

Reed, M., \& Simon, B. (1975). Methods of modern mathematical physics. Volume 2: Fourier analysis, selfadjointness. New York: Academic Press.

Rovelli, C. (1996). Relational quantum mechanics. International Journal of Theoretical Physics, 35(8), $1637-1678$.

Schwartz, L. (1950-1951). Théorie des Distributions, Vol. 1 and 2 (1st ed.). Paris: Hermann \& Cie.

Streater, R. F., \& Wightman, A. (1964). PCT, spin and statistics, and all that. Boston: Addison Wesley.

Summers, S. J. (2012). A perspective on constructive quantum field theory. arXiv:1203.3991 [math-ph]. This is an expanded version of an article commissioned for UNESCO's Encyclopedia of Life Support Systems (EOLSS).

Szász, D. (1996). Boltzmann's ergodic hypothesis, a conjecture for centuries? Studia Scientiarum Mathematica Hungarica, 31, 299-322. Lecture given at the international symposium in honour of Boltzmann's 150th birthday. Vienna February 24-26, 1994. Erwin Schrödinger Institute preprint 98, May $16,1994$.

Thirring, W. (2001). J. v. Neumann's influence in mathematical physics. In M. Rédei \& M. Stöltzner (Eds.), John von Neumann and the foundations of quantum physics (Vienna Circle Institute Yearbook, Vol. 8, pp. 5-10). Alphen aan den Rijn: Kluwer.

Valente, G. (2008). John von Neumann's mathematical "Utopia" in quantum theory. Studies in History and Philosophy of Science Part B: Studies in History and Philosophy of Modern Physics, 39(4), 860-871.

von Neumann, J. (1927a). Mathematische Begründung der Quantenmechanik. Göttinger Nachrichten, 1-57. Communicated by M. Born on May 20, 1927. (Reprinted in A. H. Taub (Ed.). John von Neumann: Collected works. Vol. 1: Logic, theory of sets and quantum mechanics. New York and Oxford, 1961: Pergamon Press.) No. 9.

von Neumann, J. (1927b). Wahrscheinlichkeitstheoretischer Aufbau der Quantenmechanik. Göttinger Nachrichten, 245-272. Communicated by M. Born on November 11, 1927. (Reprinted in A. H. Taub (Ed.). John von Neumann: Collected works. Vol. 1: Logic, theory of sets and quantum mechanics. New York and Oxford, 1961: Pergamon Press.) No. 10.

von Neumann, J. (1927c). Thermodynamik quantenmechanischer Gesamtheiten. Göttinger Nachrichten, pages 273-291. Communicated by M. Born on November 11, 1927. (Reprinted in A. H. Taub (Ed.). John von Neumann: Collected works. Vol. 1: Logic, theory of sets and quantum mechanics. New York and Oxford, 1961: Pergamon Press.) No. 11.

von Neumann, J. (1930). Allgemeine Eigenwerttheorie Hermitescher Funktionaloperatoren. Mathematische Annalen, 102, 49-131. Received by the journal on December 15, 1928. (Reprinted in A. H. Taub (Ed.). John von Neumann: Collected works. Vol 2: Operators, ergodic theory and almost periodic functions in a group. New York and Oxford, 1961: Pergamon Press.) No. 1.

von Neumann, J. (1932a). Mathematische Grundlagen der Quantenmechanik. Berlin: Springer. English translation: Mathematical Foundations of Quantum Mechanics. Princeton, 1955: Princeton University Press.

von Neumann, J. (1932b). Proof of the quasi-ergodic hypothesis. Proceedings of the National Academy of Sciences, 18(1), 70-82. (Reprinted in A. H. Taub (Ed.). John von Neumann: Collected works, Vol. 2: Operators, ergodic theory and almost periodic functions in a group. New York and Oxford, 1961: Pergamon Press.) No. 12. 
von Neumann, J. (1961). The mathematician. In A. H. Taub (Ed.) John von Neumann: Collected works (Vol. 1, pp. 1-9). New York: Pergamon Press. Originally published in Works of the Mind (Vol. 1, no. 1, pp. 180-196). Chicago, 1947: University of Chicago Press.

Wightman, A. S. (1983). Hilbert's 6th problem. In F. E. Browder (Ed.), Mathematical developments arising from Hilbert Problems (Proceedings of symposia in pure mathematics, Vol. 28, pp. 147-240). Providence, RI: American Mathematical Society.

Wightman, A. S., \& Gårding, L. (1964). Fields as operator-valued distributions in relativistic quantum theory. Arkiv f. Fysik, Kungl. Svenska Vetenskapsak, 28, 129-189.

Wigner, E. (1960). The unreasonable effectiveness of mathematics in the natural sciences. Communications in Pure and Applied Mathematics, 13(1), 1-14.

Publisher's Note Springer Nature remains neutral with regard to jurisdictional claims in published maps and institutional affiliations. 